\title{
Komplikationen in der Kniechirurgie des Rheumatikers
}

\section{Complications in Knee Surgery of Rheumatic Patients}

\author{
Autoren \\ C. Biehl, U. Thormann, C. Heiß
}

Institut

Klinik für Unfall-, Hand- und Wiederherstellungschirurgie, Universitatsklinikum Giessen und Marburg Standort Giessen, Giessen

\section{Schlüsselwörter}

perioperative Komplikationen, Kniegelenk, Kniechirurgie, Rheumatoide Arthritis, Komplikationsregister

\author{
Key words \\ perioperative complications, knee, knee surgery, \\ rheumatoid arthritis, register of complications \\ Bibliografie \\ DOI https://doi.org/10.1055/s-0042-108724 \\ Online-Publikation: 19.9.2016 \\ Akt Rheumatol 2018; 43: 73-81 \\ (c) Georg Thieme Verlag KG Stuttgart · New York \\ ISSN 0341-051X \\ Korrespondenzadresse \\ Dr. Christoph Biehl \\ Klinik für Unfall- Hand- und \\ Wiederherstellungschirurgie \\ Universitatsklinikum Giessen und Marburg Standort Giessen \\ Rudolf-Buchheim-Straße 7 \\ 35385 Giessen \\ Tel.: + 49/641/98557 660, Fax: +49/641/98544609 \\ drchristoph.biehl@arcor.de
}

\section{ZUSAMMENFASSUNG}

Im Langzeitverlauf der Rheumatoiden Arthritis (RA) ist das Kniegelenk nahezu immer in das Krankheitsgeschehen mit einbezogen. Gleichzeitig sind entzündlich destruierte Kniegelenke der häufigste Grund für schwere Beeinträchtigungen der ADL (Verschlechterung der sozialen Teilhabe!!) unserer Patienten. Erschwerend kommt hinzu, dass es gegenüber Komplikationen nach therapeutischen Maßnahmen besonders anfällig ist. Diese Übersicht zeigt mögliche Komplikationen im Zusammenhang mit Interventionen auf und bietet Lösungsansätze. Jede Abteilung, die sich um diese Patienten bemüht, benötigt ein stadienadaptiertes Konzept und perioperatives Management um mögliche Risiken so gering wie möglich zu halten.

\section{ABSTRACT}

The knee joint is nearly always involved in long-term pathological processes in rheumatoid arthritis (RA). Knees with inflammatory damage are the most common cause of severe impairment of our patients' activities of daily life (deterioration in social participation!!). To make matters worse, the knee is particularly vulnerable to complications after therapy. This overview describes possible complications associated with interventions and provides solutions. Each department that cares for these patients requires a stage-adapted concept and perioperative management to minimise potential risks.

\section{Einleitung}

Das Kniegelenk, als das größte Gelenk des menschlichen Körpers, ist aufgrund seiner Komplexität gegenüber jedweden unphysiologischen Belastungen und insbesondere Komplikationen nach therapeutischen Maßnahmen besonders anfällig.

Im Langzeitverlauf der Rheumatoiden Arthritis (RA) ist das Kniegelenk, vorwiegend bilateral, in über $90 \%$ nahezu immer in das Krankheitsgeschehen mit einbezogen. Gleichzeitig sind entzündlich destruierte Kniegelenke der häufigste Grund für schwere Beeinträchtigungen der ADL (activities of daily living)(Verschlechterung der sozialen Teilhabe!!) unserer Patienten.
Wenn der Befall der Kniegelenke therapeutische Konsequenzen im Sinne vermehrter operativer Interventionen nach sich zieht, so bedeutet dies auch, dass die Wahrscheinlichkeit von Komplikationen ebenfalls steigt.

In der medizinischen Literatur treten Arbeiten über Komplikationen gegenüber der Veröffentlichung positiver Ergebnisse deutlich zurück. Sie sind aber vorhanden und beeinflussen ärztliches und therapeutisches Handeln.

Insofern ist das Benennen von Komplikationen und das Entwickeln von Strategien zur Lösung, besser noch Vermeidung dieser Ereignisse hilfreich. 


\section{Klinik}

Das klinische Bild der rheumatischen Gonarthritis ist meistens sowohl dem Patienten, als auch dem Therapeuten frühzeitig ersichtlich. Die proliferierende Synovialis führt zu einem überschießenden Aufbau von Zellschichten mit Verdickung der Kapsel und zu vermehrter Ergussbildung und somit zu einer sichtbaren Schwellung des Gelenkes. Synoviale Schwellungen imponieren in den ventralen Gelenkabschnitten meist parapatellar, dorsal als raumfordernde Bakerzyste. Begleitend und erschwerend kommt häufig eine Abflussstörung der distal gelegenen Venen und Lymphgefäße hinzu, die zu peripheren Ödemen führen.

Die Synovialitis führt zur direkten Destruktion des Knorpels durch pannöse Überwucherung und zur sekundären Schädigung der Gelenkflächen durch chondrodestruktive Enzyme (z. B. Metalloproteasen) und Schädigung der gelenkstabilisierenden Bandstrukturen durch chronische Überdehnung und infiltratives Wachstum.

Schon vor Einführung der Biologicals gab es hochakute Verläufe, die dem o.g. Bild durch ein Fehlen der Schwellung nicht entsprachen, dennoch zu einer rasch progredienten Destruktion der Gelenke führten und führen.

Merke, eine eindeutige sichtbare Schwellung ist nicht zwingende Voraussetzung für eine aktive Gonarthritis.

Kniegelenke, die trotz suffizienter Basistherapie eine Entzündungsaktivität aufweisen sollten innerhalb von 6 Wochen einem orthopädischen Rheumatologen mit der Frage des Interventionsbedarfs vorgestellt werden. Nur so lassen sich langfristige negative Folgen für das Gelenk und den Patienten vermeiden.

\section{Therapieplanung}

Die meisten rheumaorthopädisch erfahrenen und tätigen Orthopäden richten ihre Entscheidung bei der Therapie einer rheumatischen Gonarthritis an einem etablierten und praktikablen Thera- piekonzept aus, dass die Besonderheiten des Rheumapatienten berücksichtigt $[1,2]$.

Um das Risiko einer Komplikation bei Eingriffen am rheumatisch geschädigten Kniegelenk so gering wie möglich zu halten ist ein perioperatives Management unabdingbar [3]. Hierzu gehört eine dezidierte Anamnese, die neben Begleiterkrankungen und Allergien auch eine genaue Auflistung der rheumatologischen medikamentösen Therapie umfasst $[4,5]$. Aber auch die regelmäßige klinische Untersuchung aller Gelenke gehört zwingend dazu (Rehart: „treat-to-target“ aller Gelenke!).

\section{Therapiekonzept nach LDE - Stadien}

Eine Erkrankung wie die Rheumatoide Arthritis mit ihrem stadienartigen Verlauf verlangt ein angepasstes Vorgehen seitens des Orthopäden und Unfallchirurgen, indem konservative und operative Versorgungen am Kniegelenk berücksichtigt werden müssen ( Abb.1) [6].

Primär gilt in der Versorgung einer Extremität die Prämisse proximal vor distal. Je nach Befall, Destruktion und Einschränkung des Patienten können die übrigen Gelenke der Extremität eventuell ein Wechsel des Therapieplans erzwingen. So muss möglicherweise bei bekanntem destruierten Kniegelenk ein Sprunggelenk zunächst versorgt werden, um dieses Gelenk zu erhalten oder die Belastbarkeit des Beines nach Versorgung des Kniegelenkes zu gewährleisten.

Die verschiedenen Therapieoptionen unterliegen ebenfalls einem Wandel, der dem medizinischen Fortschritt geschuldet ist. Allerdings können Verfahren wieder entdeckt werden, die zwischenzeitlich als überholt galten (z. B. mod. RIAP-Verfahren am Ellenbogen)[7].

Doch trotz aller Vorsichtsmaßnahmen und Berücksichtigung aller Eventualitäten können Komplikationen auftreten und Rheumatiker haben per se ein erhöhtes Risiko für Komplikationen [8].



Abb. 1 Therapiekonzept nach LDE-Stadien Kniegelenk. 
Diese können zu verschiedenen Zeitpunkten in Bezug auf eine therapeutische Intervention vorkommen. Man unterscheidet in Bezug zum Operationszeitpunkt frühe von späten Komplikationen. Die Art und Weise der Komplikation ist darüberhinaus abhängig von dem betroffenen Gelenk und dem Eingriff.

Aufgrund der eingeschränkten Kompensationsmöglichkeiten durch die meist ebenfalls betroffene Nachbargelenke treten Probleme und Komplikationen am Kniegelenk des Rheumatikers früher und ausgeprägter zu Tage, als bei Patienten mit einer Arthrose.

Am Kniegelenk kann es zu lokalen Komplikationen aufgrund der Rheumatoiden Arthritis kommen, wie Synovialitisrezidive im ventralen Gelenkabschnitt und der dorsalen Kapsel. Daneben stehen Band- und Sehnenpathologien und die lokale Situation der Gelenkflächen und des periartikulären Knochen im Sinne von Osteonekrosen, inkl. Looser-Umbauzonen. Seltener als beim Arthrotiker kommt es zu ausgeprägten Bewegungseinschränkungen und Arthrofibrosen. Infektionen, Thrombosen mit und ohne Embolien und Schmerzen, bis hin zum CRPS I sind nicht alleinig bei Rheumatikern zu finden. Genauso wenig wie die Komplikationen nach endoprothetischem Ersatz. Periprothetische Frakturen können bei Rheumatikern mit der Komorbidität einer Osteoporose bereits bei Bagatelltraumata auftreten. Fehlpositionierte Endoprothesen führen langfristig zur Dekompression der stabilisierenden Strukturen mit Beschwerden im gesamten Bein mit Einschränkung der Mobilität.

Des Weiteren können sich Komplikationen am Kniegelenk darstellen, die ihren Ausgang an anderer Stelle des generalisiert betroffenen Rheumatikers nehmen. Hierzu zählen vor allem die Problematik der Achsabweichung und Fehlbelastung durch eine insuffiziente Muskulatur.

\section{Synovialitis Rezidive}

Bei persistierender Synovialitis im Kniegelenk, meist vergesellschaftet mit einer dorsalen Bakerzyste ist die operative Entfernung der entzündlich veränderten Schleimhaut indiziert. Je nach Ausmaß der Bakerzyste und dem ventral befindlichen Synovialgewebe wird zunächst die Zyste entfernt, das Gelenk synovialektomiert und die dorsale Kapsel verschlossen, um in einer zweiten Operation das Kniegelenk ventral zu versorgen. Dies geschieht arthroskopisch, anschließend sollte eine Radiosynoviorthese (RSO) erfolgen, um etwaige Synovialzellnester zu veröden [9-11].

Inzwischen ist die offene, radikalere Synovialektomie meist nur noch speziellen Befunden, wie einer bereits fibrotisch veränderten Synovia vorbehalten. In gleicher Sitzung ist die Adressierung der Bakerzyste möglich.

Nach arthroskopischer oder offener Synovialektomie am Kniegelenk kann es bei einem hohen Synovialitis-score des Gewebes zu einem Wiederaufflammen der Entzündung im Gelenk mit erneuter Synovialitis kommen [12]. Häufig beruhen Rezidive auf mangelnder Radikalität der Operation oder auf einer zeitlichen Verzögerung der Folgeoperation, sodass das verbliebene Gewebe weiter proliferieren kann.

Bei der Terminierung der ersten Operation muss die nachfolgende OP und die bei arthroskopischer Synovialektomie notwendige RSO im jeweiligen 6-Wochen-Abstand fest eingeplant werden.

\section{Dorsale Synovialzysten}

Je nach Größe und Füllungszustand der dorsalen Zysten können diese spontan rupturieren und Synovialgewebe entlang der Muskelfaszien im Kompartiment verteilen, aber auch eine intraoperative Verschleppung von Gewebe ist möglich. Folglich sollte vor einer operativen Intervention einer Bakerzyste eine Abklärung mithilfe einer Sonografie erfolgen, um das Ausmaß der Zyste einschätzen zu können. Die MRT ist speziellen Fragestellungen, wie der differenzialtherapeutischen Abklärung einer pigmentierte villonodulären Synovialitis vorbehalten (Hämosiderinablagerungen in der Zellschicht).

Akute Rupturen werden nicht selten als Thrombose oder Hämatome fehlgedeutet. Auch bei offensichtlicher Ruptur sollte dennoch der Ausschluss einer Thrombose und eines Kompartmentsyndroms erfolgen, um den Patienten durch weitreichendere Komplikationen zu gefährden.

Versprengtes Gewebe kann selbst proliferativ aktiv sein und „Satellitenzysten“ bilden, die ihrerseits zu Beschwerden führen können.

Bei der operativen Entfernung der dorsalseitigen Zysten und Schleimhaut können neben den Muskeln wenn auch selten die tiefen Leitungsbahnen und Rami des N. cutanaeus femoris in Mitleidenschaft gezogen werden.

Komplikationen können durch sorgfältige Präparation der Zyste und der dorsalen Kapselanteile mit gründlichem Verschluss, sowie entsprechender Drainage minimiert werden.

\section{Band- und Sehnenschäden}

Durch die synovialen Veränderungen kommt es zu Überdehnungen der Seitenbänder, zu infiltrativem Wachstum in die Kreuzbänder mit Aufspleißung und somit zu indirekten und direkten Bandläsion. Die Folge sind eine Instabilität im Kniegelenk und Überlastung des Gelenkes und der gesamten Extremität. Mit der gestörten Kinematik entwickelt sich im Gegensatz zum Arthrotiker eher eine lateral betonte Knorpeldestruktion und Gonarthritis ( $>$ Abb. 2).

Nach offenen Eingriffen am Kniegelenk kann es zu Schäden der Sehnen, insbesondere der ventralen Quadrizeps- und Patellarsehnen kommen. Rupturen der Quadrizepssehne treten anschließend meist nach forcierter Mobilisation des operierten Kniegelenkes auf, aber auch als Spontanrupturen nach Bagatellverletzungen ( $\triangleright$ Abb. 3).

Häufig erfolgte hierbei der offene Zugang zum Kniegelenk transligamentär mit Irritation der schon vorgeschädigten Insertion des ventralen parapatellaren Band- und Sehnenapparates, in Verbindung mit einer ausgedehnten Denervation der Patella. Durch die immunsupprimierenden Medikamente der rheumatologischen Therapie werden die Reparaturmechanismen bei der Sehnenheilung irritiert, bis unterdrückt [13]. Folglich ist die Heilung eingeschränkt und verzögert, was bei der Präparation berücksichtigt werden muss.

Neben dem alternativen Subvastus-Zugang erlaubt auch die schonende Denervation des kranialen Patellapols eine Schonung der patellaren Insertion [14]. Bei knochennahen Rupturen erfolgt die transossäre Augmentation der Sehne mit Nahtankern und anschließende Versorgung mit einem Tutor und Begrenzung der Flexion auf rund $30^{\circ}$ für 6-8 Wochen. Bei wiederholten Rupturen bleibt als ultima ratio die Patellektomie mit ausgedehnter ventralen Kapsuloplastik und nachfolgender Ruhigstellung, oder sogar die Arthrodese des Gelenkes. 


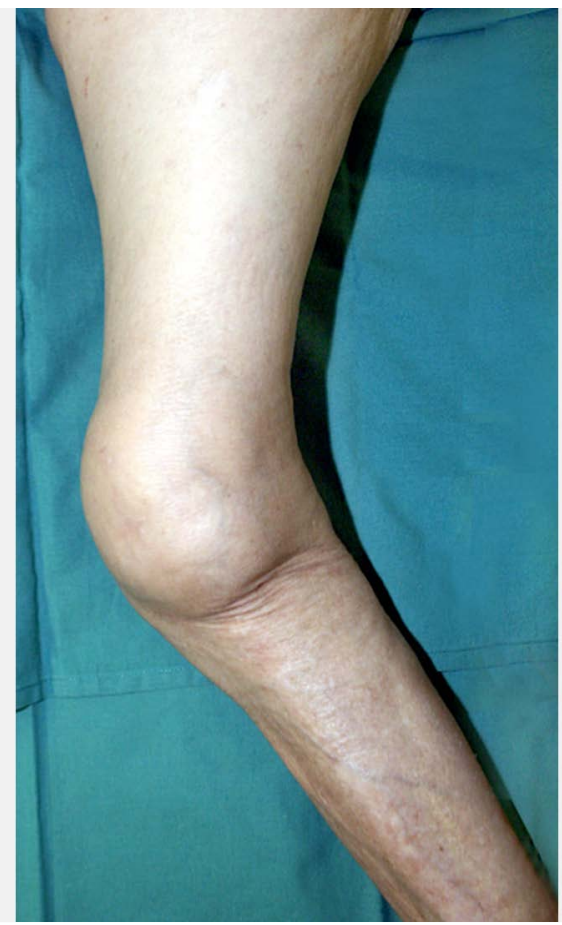

Abb. 2 Klinisches Bild eines rheumatischen Genu valgum.

\section{Knorpeldestruktionen}

Die inflammatorisch veränderte Synovia schädigt auf verschiedenen Mechanismen den Knorpel. Je nach Entzündungsaktivität kann es zu einer rasch progredienten Destruktion der Gelenkflächen kommen [12]. Zerstörter Knorpel führt im Gelenk zu Druckspitzen, die der Organismus durch einen vermehrten Aufbau von (minderwertigem) Knochen und Faserknorpel zu kompensieren versucht. Chondroprotektive oder -rekostruktive Verfahren beschränken sich beim Rheumatiker auf die Mikrofrakturierung. Für Doppel-Osteotomien, wie sie durch Benjamin für das Kniegelenk vorgeschlagen und lange Zeit vor allem für jüngere Rheumatiker praktiziert wurden, gibt es heute praktisch keine Indikation mehr [15].

\section{Knochenschäden}

Bereits in frühen Stadien der Einbeziehung eines Gelenkes in das rheumatische Krankheitsgeschehen (LDE 0-1), lässt sich eine periartikuläre rarefizierte Knochenstruktur nachweisen. Diese tritt bevorzugt an der Grenzlamelle auf. Mehr noch als bei Rheumatikern zeigen Psoriasispatienten das bunte Bild von räumlich nahen osteonekrotischen und osteoklastischen Bezirken im gelenknahen Knochen. Vitamin-D-Mangel kann zu gelenknahen Looser-Umbauzonen führen. Therapeutisch bleibt neben der Entlastung und Kapsel-Bandprotektion mittels Orthese eine Vitamin-D-Substitution.

Komplikationen bei Veränderungen des Knorpels und gelenknahen Knochens und der resultierenden Kinematikänderung, sowie nach operativen Eingriffen sind in der Gefahr der Bewegungseinschränkungen und Arthrofibrose zu sehen.

Drohen doch bei Kontrakturen oder endgradigen Bewegungseinschränkungen aufgrund gut gemeinter Schonung und dem Aus-

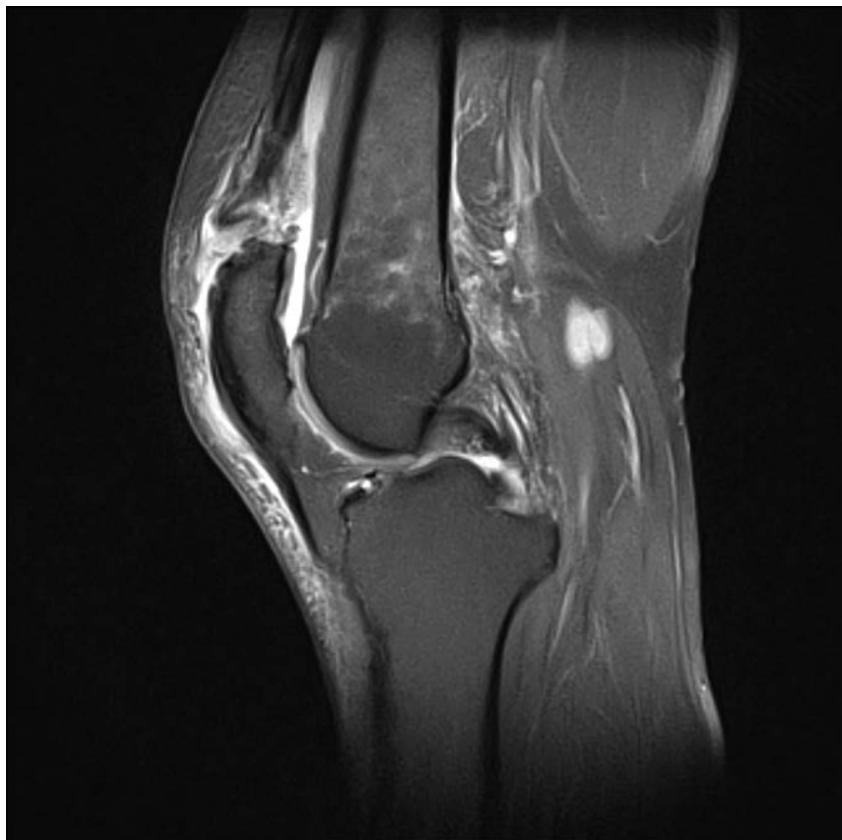

Abb. 3: Distale Quadrizepsruptur, suprapatellar, MRT seitlich,

T2-Gewichtung.

lassen des gesamten Bewegungsraums persistierende Probleme und Schmerzen paraartikulär bis hin zu Beeinträchtigungen der Gesamtmobilität der Patienten. Bei persistierenden Bewegungseinschränkungen lohnt der Blick auf die Gesamtstatik des Patienten, da häufig hierdurch Kontrakturen an anderer Stelle ausgeglichen werden. Eine entsprechende Korrektur wird unter diesen Voraussetzungen zu Rezidiven neigen, um die Gesamtmobilität des Patienten nicht zu gefährden [16].

\section{Endoprothesen}

Bei fortgeschrittener Destruktion der Gelenkflächen ist die endoprothetische Versorgung indiziert. Mit Ausnahme einiger Zentren wird die Versorgung eines rheumatischen Kniegelenkes mit einer unikondylären Prothese nicht durchgeführt, da die meisten Operateure dies mit dem Hinweis des generalisierten Krankheitsgeschehens mit Einbeziehung des gesamten Gelenkes ablehnen. Dierkes et al. konnten in ihrer retrospektiven Untersuchung zeigen, dass die Möglichkeit einer unikompartimentellen Versorgung gegeben ist, wenn gleichzeitig eine radikale Synovialektomie erfolgt [17]. Der Oberflächenersatz gilt jedoch auch bei Rheumatikern als Goldstandard.

Komplikationen um die endoprothetische Versorgung können zumeist intra- und postoperativ auftreten.

Sehr häufig finden sich in inflammatorisch destruierten Gelenken Zonen stark sklerosierten Knochens neben hochgradig osteoporotisch rarefizierten Bereichen. Hierbei kann es bereits beim Zurichten des Knochens zu Frakturen oder zu knöchernen Ausrissen der Seitenbandansätze kommen. Diese werden intraoperativ refixiert anschließend das Kniegelenk mit einer temporären Orthese versorgt. In fortgeschrittenen Fällen muss auf eine achsgeführte 
Prothese zurück gegriffen werden. Aber auch nach Lastaufbau kann es im Verlauf zu einer periprothetischen Ermüdungsfraktur kommen, bei der der sofortige Wechsel der Prothese nicht erforderlich ist, sondern die stabile Osteosynthese eine sinnvolle Therapieoption darstellt. Gleichwohl gilt es die Gesamtsituation mit den Kompensationsmöglichkeiten zu beachten.

Auch bei Rheumatikern kommt gelegentlich die Frage nach der endoprothetischen Versorgung der retropatellaren Gelenkfläche auf. Diese ist, zumindest in Europa, eine Rarität, da die oben beschriebene Knochenproblematik auch und im Besonderen für die Patella zutrifft. Hier besteht die Gefahr der frühzeitigen Lockerung mit Bruch der Patella und dem Freisetzen von Zementanteilen, die Schäden im PE der Prothese hervorrufen können und so die Standzeit verkürzen. Als Alternative bleibt neben der Denervation des Ramus infrapatellaris und der Patella das Überziehen der patellaren Gelenkfläche mit einem Kapsellappen als Patella-RIAP [2].

Bei rheumatischen Kniegelenken sollte bei der Implantation einer Prothese die gegenüber Arthrotikern geänderte Kinematik der Band- und Sehnenstrukturen im Langzeitverlauf beachtet werden. Rheumatiker neigen aufgrund der synovialen Dehnungszustände der Kapsel eher zum nachträglichen Lockern der Bandverbindungen, sodass primär eine straffere Implantation des Oberflächenersatzes anzustreben (Ausnahme unikondyläre Prothese) ist. Dies wiederum bedingt direkt postoperativ eine erhöhte Beachtung der vollen Extension im Gelenk.

Bei bereits intraoperativ kritischer Bandführung sollte eine bandentlastende, (teil)gekoppelte Prothese erwogen werden, insbesondere wenn die postoperative Versorgung nicht ausreichend in Besonderheiten des Rheumatikers geschult ist [7].

\section{Periprothetische Komplikationen}

Bei und nach der Implantation von Knieprothesen kann es zu Komplikationen kommen, die bereits weiter oben erwähnt wurden, aber bei dieser Versorgung relativ häufiger auftreten. Endoprothesen-pflichtige Kniegelenke sind an Knochen, Knorpel und periartikulären Weichteilen weitaus schwerer geschädigt, als Knie, die gelenkerhaltend operativ versorgt werden können. Insofern verwundert dies nicht.

Eine der Hauptfragen von Patienten ist die nach der Haltbarkeit der Prothese. In den letzten Jahren konnten hier Fortschritte bei den tribologischen Eigenschaften der Prothesen erreicht werden. Dennoch ist und bleibt insbesondere bei Rheumatikern für die Überlebensdauer in situ die komplette und akribische Synovialektomie bei der Primäroperation entscheidend [18].

Kontrakturen der Seitenbänder können mittels femoraler Osteotomie und Verschraubung korrigiert werden [7], gleichwohl reichen bereits Bagatelltraumata, um vorgeschädigte Weichteilstrukturen zu schädigen ( $\triangleright$ Abb. 4 ).

Operativ bleibt der Versuch der Rekonstruktion der Strukturen, oder der Wechsel auf ein achsgeführtes Implantat. Erstere Versorgung wird man bei jüngeren Patienten anstreben, um sich nicht frühzeitig die Rückzugsmöglichkeiten im Langzeitverlauf der RA zu berauben.

Langjährige rheumatoide Arthritis bedingt eine reduzierte Knochendichte, die durch die steroidale Basistherapie oft weiter reduziert wird und zu einer deutlich erhöhten Frakturgefahr führt. Ins-

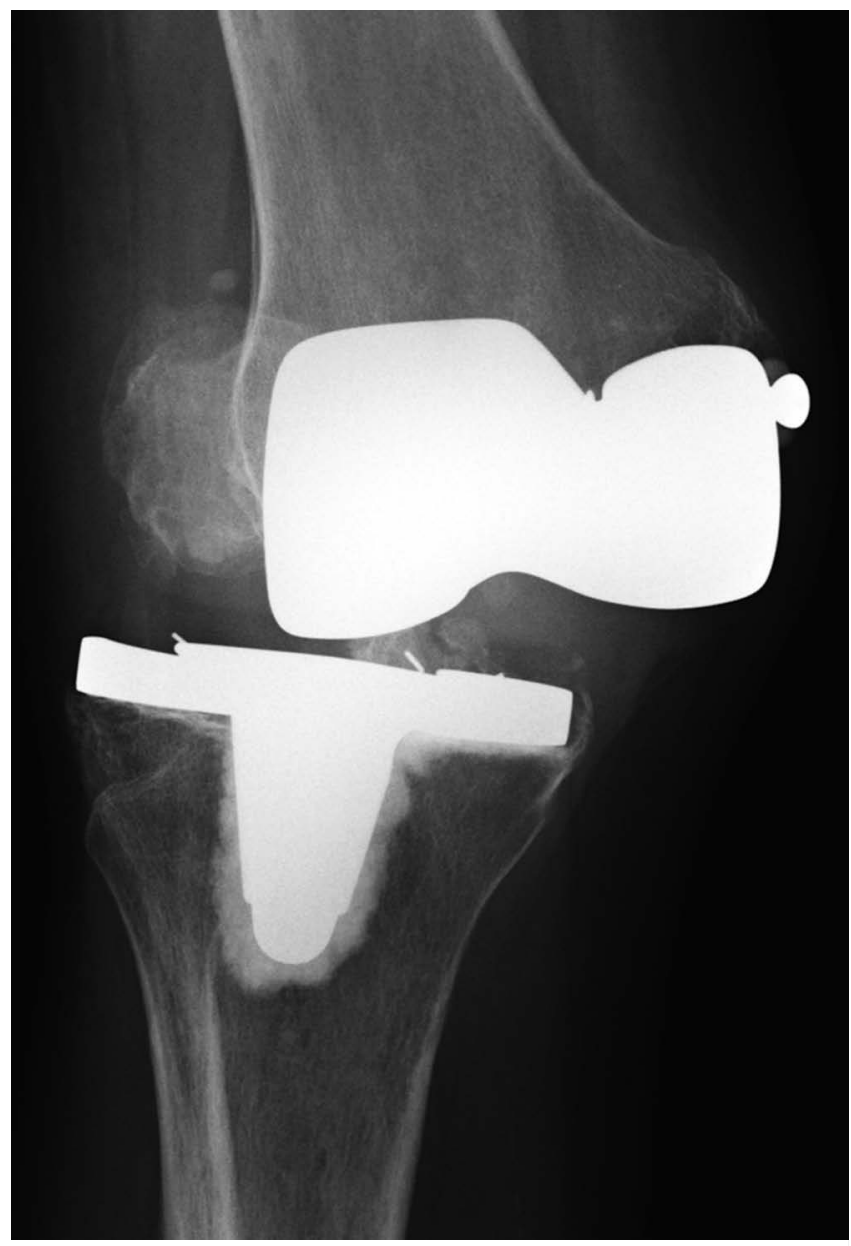

- Abb. 4 schwere Bandschäden mit instabilem Kniegelenk nach Bagatelltrauma.

besondere die periartikulären knöchernen Strukturen sind anfällig für osteoporotische Veränderungen und weisen eine erhöhte Frakturgefahr auf. Bei eingetretener Fraktur muss primär die Stabilität der Endoprothese geklärt werden, da sich hieran die operative Versorgung ausrichtet. Entweder kann das Gelenk belassen werden und es erfolgt die Frakturversorgung mittels Osteosynthese (winkelstabile Platte, retrograder Femurnagel) oder durch Wechsel der Knieprothese auf ein achsgeführtes Model ( $\triangleright$ Abb. 5,6). Gleichzeitig muss die schlechte Knochenqualität und verzögerte Heilung bei der Versorgung und Heilung berücksichtigt werden, drohen doch Materialermüdung und Refrakturen ( $\mathbf{A} \mathbf{b b}$. 7-10). Eine Versorgung mit einem Fixateur extern, genauso wie eine temporäre Draht-Extension muss kritisch hinterfragt werden, da neben der möglichen schlechten Verankerungsmöglichkeit im geschädigten Knochen auch die Hautschädigung und Infektgefahr bei immunsupprimierten Patienten erhöht ist. Wenn möglich sollte eine solche temporäre Anlage vermieden werden.

\section{Störungen der Achskinematik}

Wie Eingangs angerissen kann es im Achsorgan Bein zu Komplikationen kommen, die ihren Ursprung nicht durch Veränderungen 


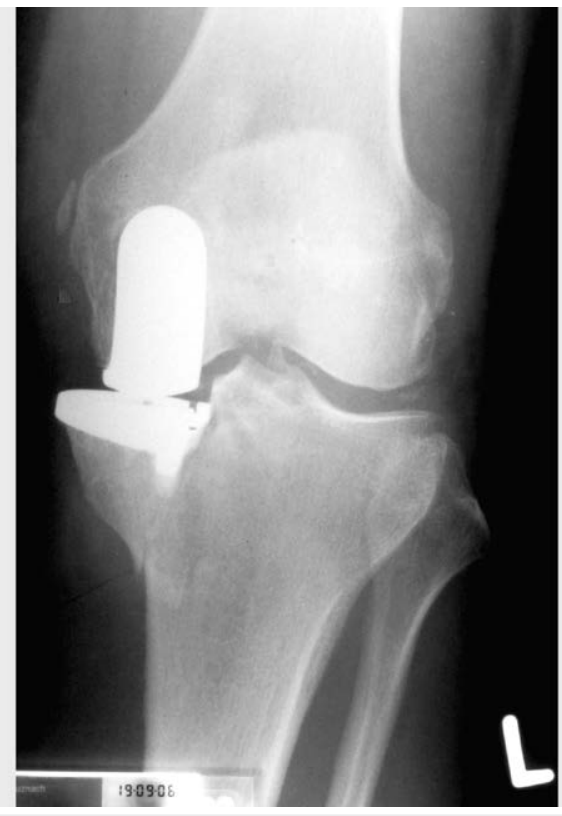

- Abb. 5 med. Knieprothese mit Tibiakopffraktur, Osteosynthese mittels winkelstabiler Platte.

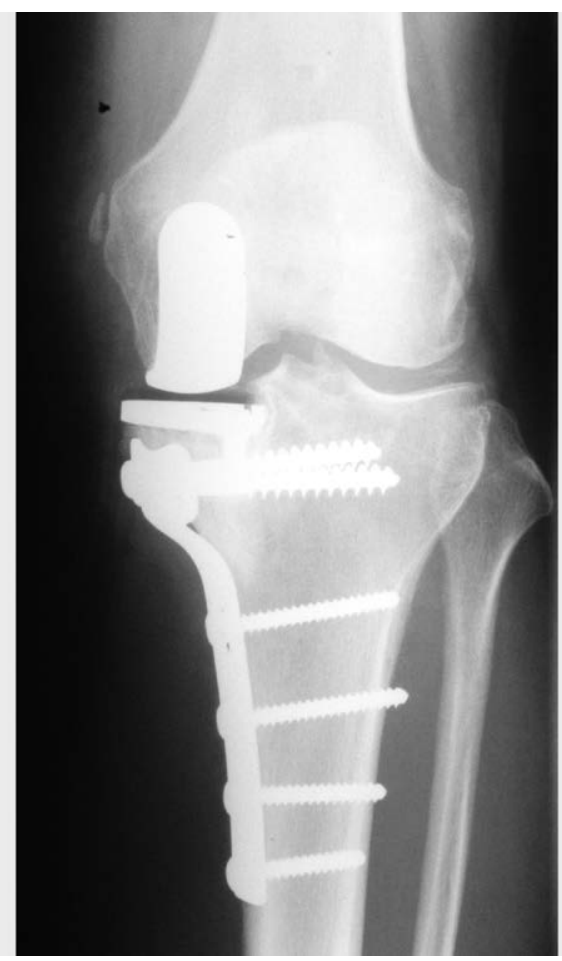

Abb. 6 Osteosynthese mittels winkelstabiler Platte bei Knie Abb. 5.

am Kniegelenk haben. Das Knie ist nur Erfolgsorgan der Störung. So können Muskelinsuffizienzen gluteal mit einer veränderten Statik und Belastbarkeit der Hüfte zu Rotationsproblemen um das Knie mit Veränderung des Patellaalignments führen ( $\triangleright$ Abb. 11). Auch Veränderungen des Sprunggelenks können zu Problemen und Schmerzen im Kniegelenk führen. Dennoch sollte immer eine sau-

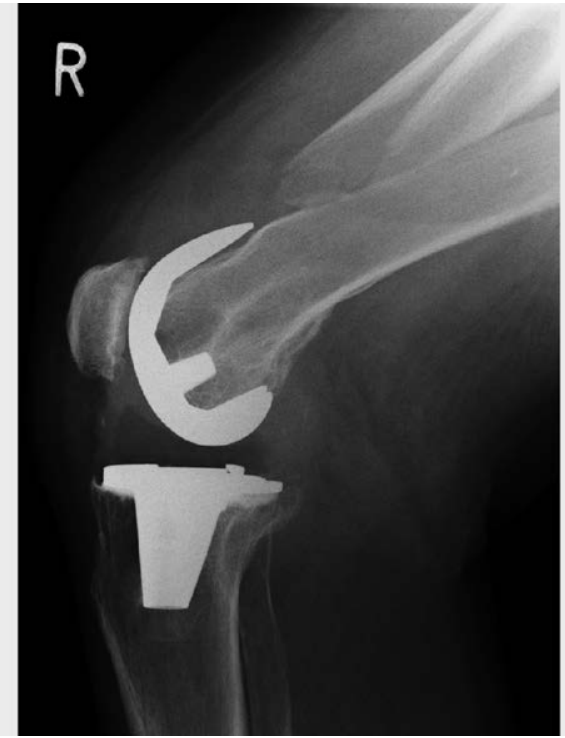

- Abb. 7 Komplikationen bei endoprothetischer Versorgung Oberflächenersatz mit distaler Femurfraktur.

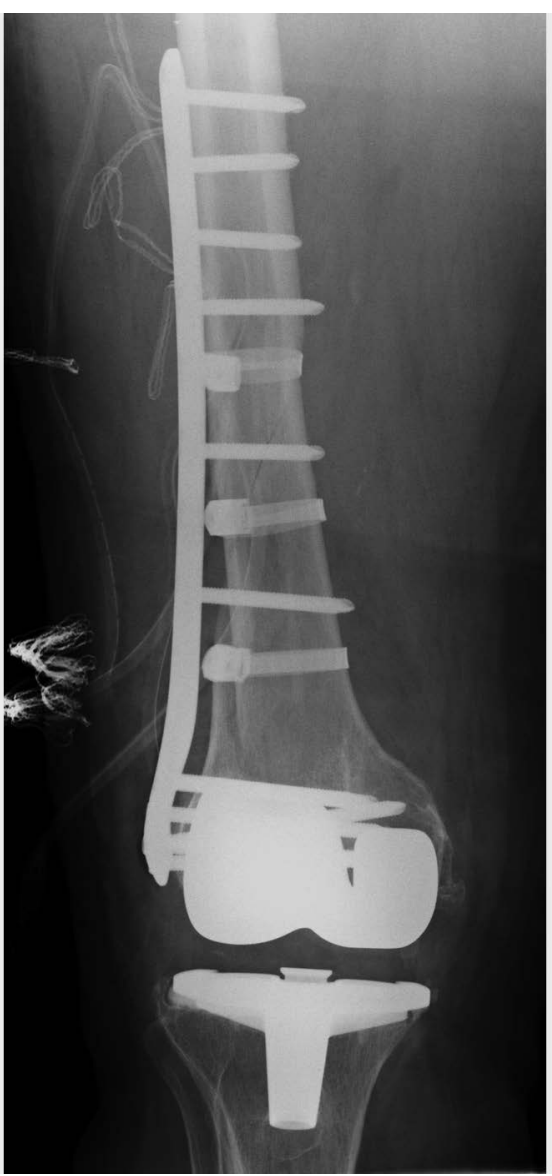

- Abb. 8 Osteosynthese nach distaler Femurfraktur von Pat. > Abb. 7.

bere Diagnostik und Abklärung der geklagten Beschwerden erfolgen. Hier bietet sich eine CT-basierte Rotationsbestimmung der Prothesenposition an [19]. 


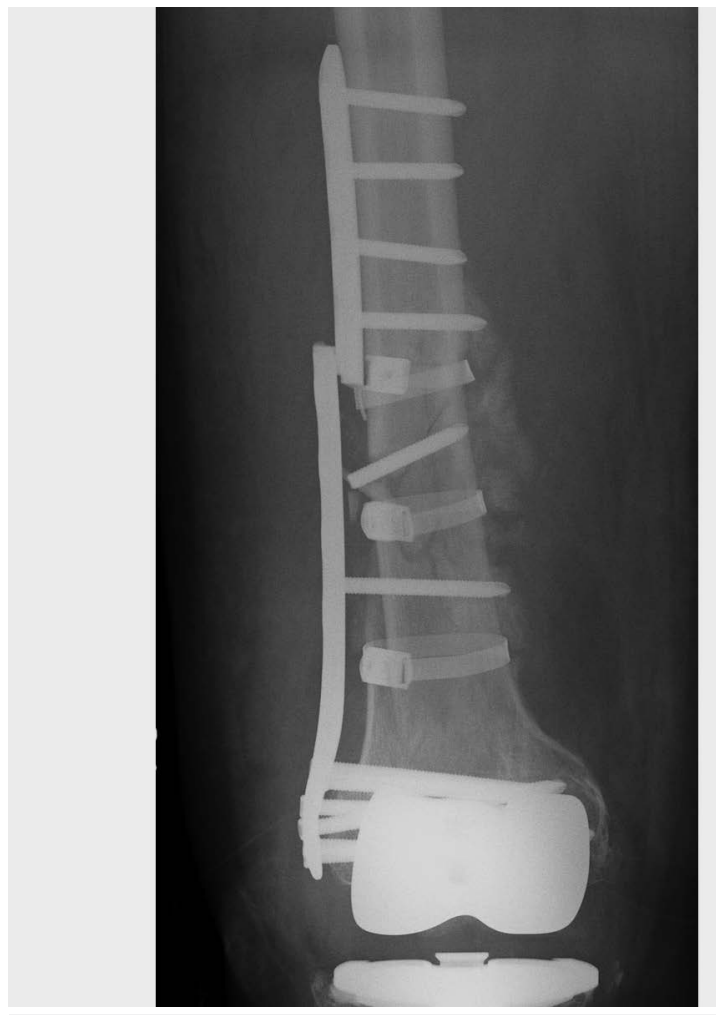

- Abb. 9 Plattenbruch als Komplikation nach Osteosynthese bei endoprothetischer Versorgung Oberflächenersatz mit distaler Femurfraktur.

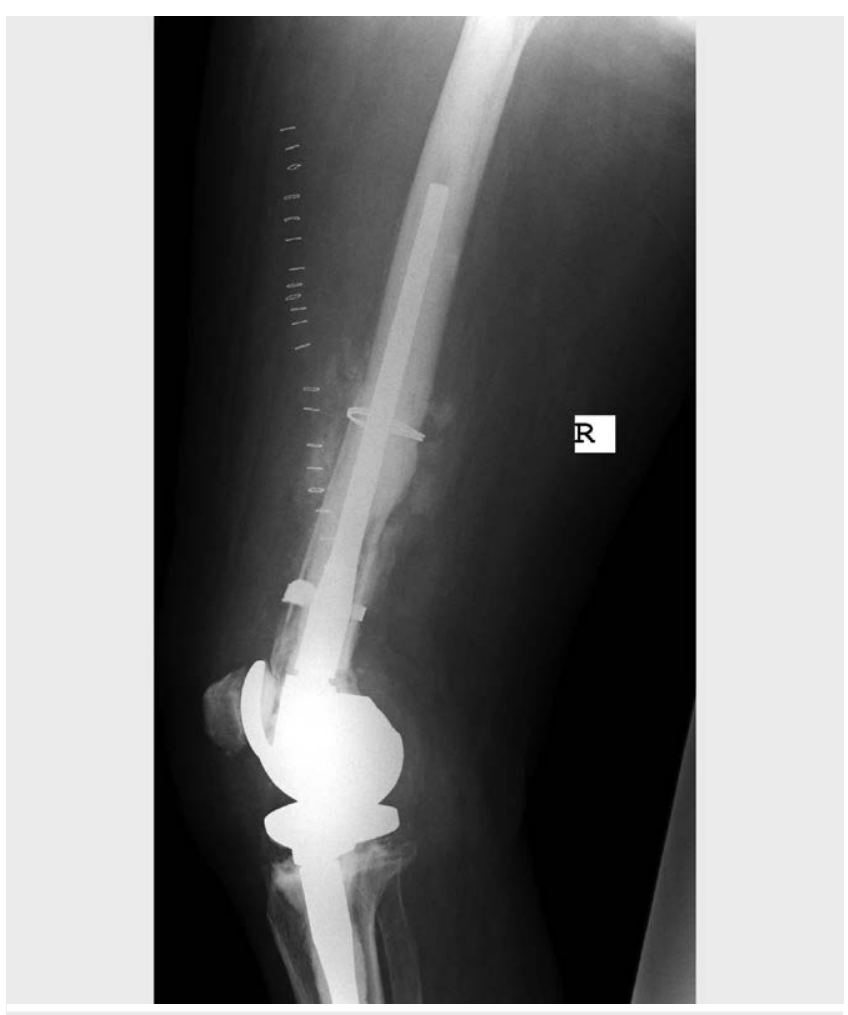

- Abb. 10 Wechsel auf Achsgeführtes Kniegelenk nach Fraktur, Osteosythese und Plattenbruch ( $\triangleright$ Abb. 7-9).

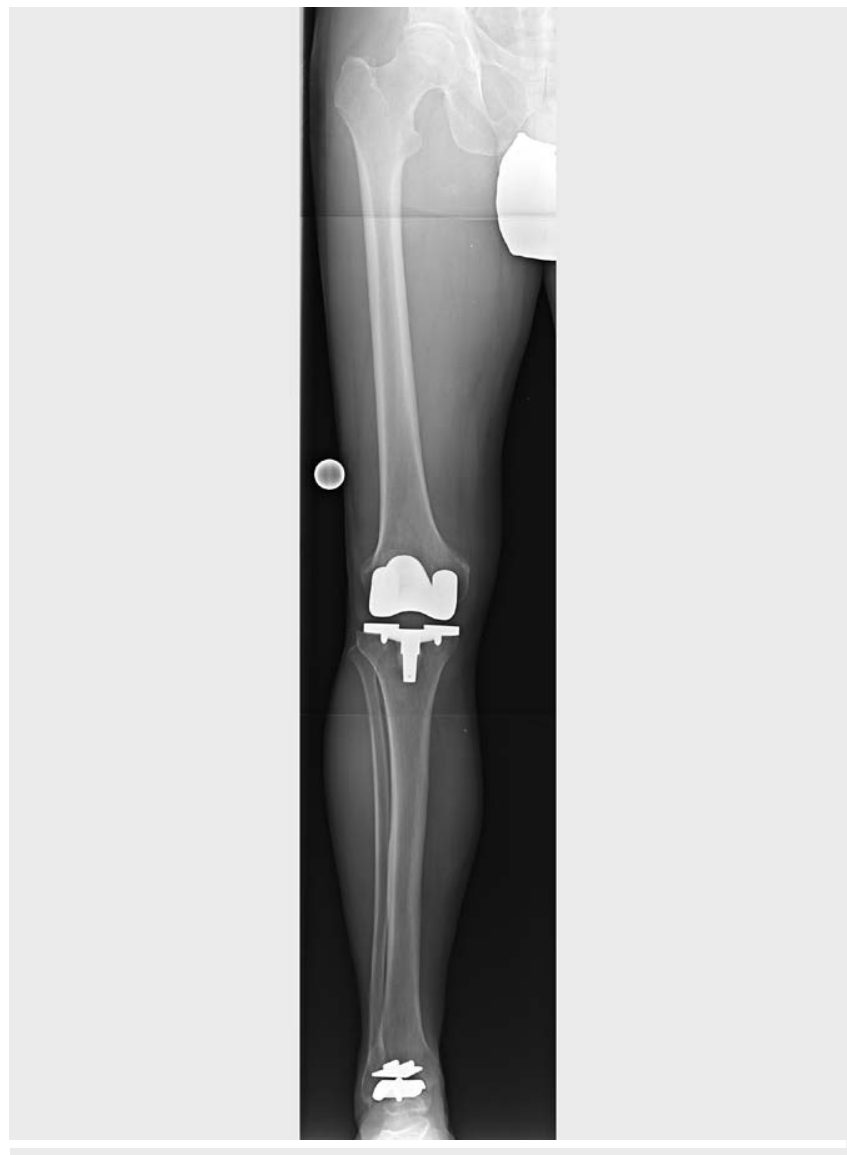

- Abb. 11 parapatellare Beschwerden bei Glutealinsuffizienz, CTAuswertung erfolgt (data not shown).

\section{Schmerzsyndrome und CRPS}

Postoperativ stehen meist Schmerzen und Bewegungseinschränkungen oder eine postoperative Schubsituation im Vordergrund, die für die Patienten eventuell belastender sind. Die Schubsituation tritt meist einige Tage nach der Operation auf, wenn die perioperative Gabe von Schmerzmitteln oder Kortison im Rahmen des Schemas reduziert wird (Rebound-Effekt). Ein verlangsamtes Ausschleichen kann die Situation verbessern. Bei einzelnen Patienten muss das pausierte Biological früher als gewünscht gegeben werden, um die rheumatische Aktivität wieder in den Griff zu bekommen. Inwieweit hier die perioperative Gabe von Biologicals in Zukunft modifiziert werden kann, soll das von der DGORh initiierte Komplikationsregister beantworten helfen [20].

Schmerzen, die mit der Operation in Zusammenhang stehend, sind meist unmittelbar danach vorhanden. Der Rheumakranke kann die Schmerzqualität meist sehr gut von den üblichen entzündlich bedingten Schmerzen unterscheiden. Schmerzen, die auf eine entsprechende Pharmakotherapie nicht oder nur unzureichend ansprechen, können sich zu einem ernsten Problem für Patient und Therapeuten ausweiten. Schmerzkatheter mit der Möglichkeit der Booster Gabe bei einer einstellbaren Basisrate sind inzwischen weit verbreitet, allerdings ist der Einsatz häufig auf die ventralen Gelenkabschnitte begrenzt, die dorsalen Bereiche von Kapsel und verkürzten Sehnen und Bändern werden nicht erreicht. 
Rheumatiker sind für ein CRPS I besonders gefährdet, da entsprechende Beschwerden leicht übergangen werden. Die Beschwerden können als tophische, motorische, sensorische oder autonome Störungen imponieren: Anfang oft unspezifisch weist der M. Sudeck häufig einen stadienartigen Verlauf mit nachfolgender Dystrophie und Atrophie über. Entscheidend für die Prophylaxe und Therapie eines CRPS I ist die suffiziente perioperative antiinflamatorische und antiphlogistische Therapie bereits in der Frühphase.

Im längeren postoperativen Intervall sind auf das Kniegelenk projizierten Schmerzen der Hauptgrund für Patientenunzufriedenheit [21]. Manche Patienten berichten über ventrale parapatellare Knieschmerzen. Hier ist neben der genauen Anamnese das Aufzeigen des Schmerzorts durch den Patienten häufig weiterführend.

Diffuse flächig angegebene, oder auch bandförmige Beschwerden im Bereich des Tibiakopfes lassen auf eine Problematik des Streckapparates, des Lig. patellae und der Patella selber schließen. Meist besteht gleichzeitig eine endgradige Streckhemmung, die die ventralen Strukturen der Proprio- und Nozizeptoren des Kniegelenkes permanent aktiviert und die Bandstrukturen überlastet. Eine Verbesserung der aktiven Streckung führt zur Reduktion des retropatellaren Anpressdrucks und somit zur Schmerzreduktion.

Punktuelle Beschwerden lassen auf eine Irritation des R. infrapatellaris schließen, der beim medialen Zugang zum Kniegelenk tangiert oder bewusst aufgesucht und denerviert wird. Neurale Beschwerden lassen sich durch eine lokale Infiltration mit Lokalanästhetika verifizieren. Anschließend kann der Nerv entweder mit Infiltrationen mit $70 \%$ Alkohol oder operativ denerviert werden.

Prinzipiell lassen sich ventrale Kniebeschwerden nach endoprothetischer Versorgung am besten durch die Auswahl und Implantation geeigneter Knieprothesensysteme vermeiden, die den retropatellaren Anpressdruck sowohl statisch, als auch dynamisch minimieren [22].

\section{Hautschäden}

Bei der operativen Versorgung des Kniegelenkes kann es leicht zu Verletzungen der teilweise papierdünnen „Kortisonhaut“ kommen. Diese führen zu einer verzögerten Heilung und der Gefahr der Sekundärkontamination, da der natürliche Schutz der Haut nicht gewahrt werden kann. Konsequenterweise werden daher Verbände ohne Zug über die Haut angelegt. Das Entfernen des Verbandes hat ohne Schere zu erfolgen, um nicht die Haut oder gar tiefere Schichten zu verletzen.

Neben der erhöhten Vulnerabilität sind Rheumatiker auch vermehrt der Gefahr der Unterkühlung ausgesetzt. Auf eine suffiziente Wärmeregulation ist durch Einsatz von Wärmematten und Umluft-/Wärmedecken zu achten. Ein warmer Patient benötigt weniger Narkose- und Schmerzmittel, das postoperative „shivering“ und die Infektionsgefahr werden reduziert.

\section{Infektionen}

Bei Patienten mit einer unterdrückten Immunantwort sind die klassischen Zeichen einer frühen Infektion häufig nicht immer eindeutig. Infekte können einen scheinbar blanden Verlauf zeigen, der dann als generalisierte Begleitreaktion auf den operativen Stress fehlgedeutet werden kann. Andererseits kann auch eine Schubsituation eine Infektion vortäuschen oder maskieren.

Die Infektionsrate ist bei Rheumatikern deutlich erhöht, manche Autoren sprechen von bis zu $50 \%$ innerhalb von 10 Jahren $[8,23]$. Patienten mit einer Infektion sind vital gefährdet und es kann sehr schnell zu lokalen und generalisierten irreversiblen Schäden kommen.

\section{Daher ist schnelles Handeln Pflicht.}

Hierbei hat jede Abteilung ihre eigenen Abläufe, die aber alle bestimmte Punkte beinhalten. Bei Erstkontakt mit einem potentiell infektiösen Patienten gehört - neben der gründlichen Untersuchung des gesamten Patienten (Statuserhebung) - die Kontrolle der Entzündungsparameter im Blut, die Gewinnung von Blutkulturen bei vorhandenem Fieber und ein Punktat des betroffenen Gelenks zur Bestimmung von Zellzahl und Keim, inklusive Antibiogramm. Sollte sich bereits bei der Punktion makroskopisch die Diagnose einer bakteriellen Infektion bestätigen ist der Patient unverzüglich operativ zu versorgen. Intraoperativ erfolgt ein sorgfältiges Debridement mit erneuter Probengewinnung für mikrobiologische und histopathologische Untersuchung, inkl. Möglichkeit der PCR. Wenn auch die Nachweisbarkeit von Keimen mit Einführung von neueren Techniken (PCR, Sonikation) verbessert worden ist, so ist nicht immer ein Keim isolierbar [24, 25]. Bei einem endoprothetisch versorgten Gelenk erfolgt der Austausch der beweglichen Komponenten gegen neue Prothesenteile, sofern es sich um einen früh angehbaren Infekt (innerhalb von Stunden bis Tagen) handelt und die Einlage von Kathetern. Bei länger bestehendem Infekt muss die Prothese entfernt und eine Interimsprothese eingesetzt werden, um die Spannung der Weichteilstrukturen zu erhalten und keine Kontrakturen zu begünstigen, die einer Replantation nach ausgeheiltem Infekt im Wege stünden. Nach Normalisierung der Entzündungswerte kann dann die Replantation der Endoprothese mit entsprechend an die Resistenzlage angepassten Zement erfolgen. [2]

Für die Zeit der Infektion ist der Patient zu isolieren, um keine unnötige und unter Umständen lebensbedrohliche Zweitinfektion oder Keimverschleppung auf Dritte zu riskieren.

\section{Thrombose und Embolie}

Die Gefahr von Thrombosen und Embolien ist bei rheumatischen Knieoperationen erhöht, da aufgrund der Systemerkrankung Rheumatiker in ihrer Mobilität nach Eingriffen an den unteren Extremitäten stärker beeinträchtigt sind. Nach den aktuellen Empfehlungen der Expertenkommissionen der S3-Leitlinie zur Thromboseprophylaxe gehören neben einer frühzeitigen Anleitung und Hilfestellung zur Mobilisation und der medikamentösen Prophylaxe mit NMH auch weiterhin die Anwendung medizinischer Thromboseprophylaxestrümpfe (MTPS)[26]. Hier gilt die oben erwähnte Hautproblematik nicht unbeachtet zu lassen. 


\section{FAZIT}

Das Kniegelenk ist nahezu immer in das Krankheitsgeschehen bei Rheuma einbezogen.

Gleichzeitig sind entzündlich destruierte Kniegelenke der häufigste Grund für schwere Beeinträchtigungen der ADL und gegenüber Komplikationen nach therapeutischen Maßnahmen besonders anfällig.

Komplikationen sind vielfältig und können zu verschiedenen Zeiten in Bezug zur eigentlichen Operation auftreten.

Ein stadienadaptiertes Konzept und perioperatives Management sind notwendig, um mögliche Risiken so gering wie möglich zu halten.

Das Wissen um die Versorgung von Rheumatikern mit all ihren Facetten und Gefahren kommt letztlich auch NichtRheumatikern zugute.

\section{Interessenkonflikt}

Nein

\section{Literatur}

[1] ARO. Rheumaorthopädie: Steinkopff. 2005

[2] Thabe H, Brackertz D. Praktische Rheumaorthopädie: Chapman and Hall. 1997

[3] Biehl C, Jung J. Perioperative Komplikationen bei Erkrankungen des rheumatischen Formenkreises. Akt Rheumatol 2015; 40: 45-50

[4] Rehart S, Henniger M. Rheumatische Erkrankungen. Orthopädie und Unfallchirurgie up2date 2012; 7: 147-162

[5] Kruger K, Albrecht K, Rehart S et al. Empfehlungen der Deutschen Gesellschaft für Rheumatologie zur perioperativen Vorgehensweise unter Therapie mit DMARD und Biologicals bei entzündlich-rheumatischen Erkrankungen. Zeitschrift fur Rheumatologie 2014; 73: 77-84

[6] Larsen A, Dale K, Eek M. Radiographic evaluation of rheumatoid arthritis and related conditions by standard reference films. Acta radiologica: diagnosis 1977; 18: 481-491

[7] Rehart S, Sell S, Arbogast M et al. Expertise Orthopädische Rheumatologie. Thieme; 2015

[8] Schöniger A, Henniger M, Rehart S. Gelenkinfektionen in der orthopädischen Rheumatologie. OUP 2013; 2: 396-399

[9] Chalmers PN, Sherman SL, Raphael BS et al. Rheumatoid synovectomy: does the surgical approach matter? Clinical orthopaedics and related research 2011; 469: 2062-2071
[10] Beil FT, Ruther W. Indications and contraindications for radiosynoviorthesis. Zeitschrift fur Rheumatologie 2015; 74: 780-785

[11] Carl HD, Swoboda B. Effectiveness of arthroscopic synovectomy in rheumatoid arthritis. Zeitschrift fur Rheumatologie 2008; 67: 485-490

[12] Krenn V, Morawietz L, Burmester GR et al. [Synovialitis score: histopathological grading system for chronic rheumatic and non-rheumatic synovialitis]. Zeitschrift fur Rheumatologie 2005; 64: 334-342

[13] Nowotny J, Kasten P. Physiologie der Sehnenheilung. Orthopädie und Unfallchirurgie up2date 2015; 10: 75-87

[14] Wirth C], Kerschbaumer F, Weise KU. Operative Zugangswege in Orthopädie und Traumatologie: Begründet von Rudolf Bauer. Fridun Kerschbaumer und Sepp Poisel. Thieme; 2013

[15] Benjamin A. Double osteotomy for the painful knee in rheumatoid arthritis and osteoarthritis. J Bone Joint Surg Br 1969; 51: 694-699

[16] Biehl C, Kappl S, Rehart S. Operative Therapie an den Gelenken bei der Spondyloarthritis. Aktuelle Rheumatologie 2013; 38: 104-108

[17] Dierkes B, Oda A, Thabe H. Lang- und mittelfristige Ergebnisse nach unikondylärer Schlittenprothese am Kniegelenk bei Patienten mit RA. 37. Kongress der Deutschen Gesellschaft für Rheumatologie e. V., Köln: 2009

[18] Fink B, Berger I, Siegmüller C et al. Recurring synovitis as a possible reason for aseptic loosening of knee endoprostheses in patients with rheumatoid arthritis. The Journal of Bone and Joint Surgery 2001; 83: 604-608

[19] Werking K, Böhme N. Einsatz der Computertomografie in orthopädischen Messverfahren. Radiopraxis 2013; 6: 143-152

[20] Kostuj T, Rehart S, Matta-Hurtado R et al. Pilot study to the registry for complications of the German Society for Surgery in Rheumatic-Diseases (DGORh) - register's method and evaluation of the first 1.000 patient's data. in print

[21] Thiele K, Fussi ], Perka C et al. The Berlin diagnostic algorithm for painful knee TKA. Der Orthopade 2016; 45: 38-46

[22] Thabe H, Dafferner-Franzmann M, Stening J. Auswirkungen verschiedener konstruktiver Prothesenmerkmale auf Langzeitergebnisse. Akt Rheumatol 2014; 39: 130-134

[23] Unger M, Winkler S.. Infektionen und Rheuma. rheuma plus 2014; 13: 13-16

[24] Trampuz A, Piper KE, Hanssen AD et al. Sonication of explanted prosthetic components in bags for diagnosis of prosthetic joint infection is associated with risk of contamination. Journal of clinical microbiology 2006; 44: 628-631

[25] Renz N, Perka C, Trampuz A. Management of periprosthetic infections of the knee. Der Orthopade 2016; 45: 65-71

[26] Encke A, Haas S, Kopp I. S3-Leitlinie Prophylaxe der venösen Thromboembolie (VTE). In AWMF; editor. 2015 